\title{
INTERVISTA A ANTONELLA ANEDDA
}

\author{
Vera Lúcia de OLIVEIRA*
}

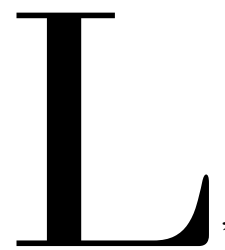

'intervista che segue è stata realizzata nell'ambito degli incontri "Poesia a Palazzo dei Priori”, di Perugia, che il Gruppo di Sperimentazione e Ricerca Poetica Il Merendacolo ha organizzato dal 1989 al 2009. Più precisamente, è stata fatta il 7 dicembre del 2000, dopo un'intensa serata in cui Antonella Anedda ha parlato della sua poesia e ha letto versi della raccolta Notti di pace occidentali (Donzelli Poesia, 1999), allora pubblicata da poco.

Queste e altre interviste da me fatte a vari poeti italiani contemporanei, come Andrea Zanzotto, Mario Luzi, Franco Loi, Valerio Magrelli, Paolo Ruffilli, Gianni D'Elia, Vivian Lamarque, Maurizio Cucchi e Giuseppe Conti, sono state pubblicate nella rivista brasiliana Insieme (São Paulo, dal numero 7 al 9, 1998-2001).

* Università degli Studi di Perugia 
Quando hai cominciato a scrivere? La scoperta della poesia è il risultato di un processo, o è avvenuta per rivelazione, per illuminazione?

Ho iniziato a scrivere presto, ma ho pubblicato le prime poesie tardi, dopo i trent'anni. La "scoperta della poesia" come tu la chiami è avvenuta attraverso la lettura verso i dodici, tredici anni di una poesia di Aleksandr Blok. Ricordo che quei versi "Ha portato il vento di lontano..." hanno avuto su di me l'effetto di uno spalancamento. C'era una porta tra il paesaggio esterno e quello interiore che a volte la poesia poteva aprire. C'era uno spazio con buone correnti, dove mi potevo mettere come un uccello o un pesce. Al liceo ho letto i classici alla luce di questo spalancamento: l'aria che trema di claritate di Cavalcanti aveva alle spalle il Cantico del Pentateuco e davanti Blok.

Qual è il tuo percorso, che poeti hai letto, che scrittori ti hanno segnato?

L'elenco è complicato e naturalmente cambia un po' nel tempo. A caldo: Dante, Foscolo, Puskin, Hopkins, Mandel'štam, Cvetaeva, Kavafis, Gertrud Kolmar, Zbignew Herbert. Ho amato e amo moltissimo Cecov e Dostoevskij (soprattutto da L'Idiota ai Fratelli Karamazov) e Leskov, e dei contemporanei mi piacciono Victor Pelevin e Ludmilla Ulickaja. Sulla corrente di Blok ho letto subito dopo Guerra e Pace di Tolstoj e Le anime morte di Gogol. Ero giovane, ma credo che questa lettura mi abbia dato la misura del respiro, l'importanza che ha nella scrittura l'ampiezza. Da allora non temo la solitudine: mi basta aprire un romanzo... Però ci sono molti altri scrittori, non solo poeti o romanzieri, che penso mi abbiano segnato: Flaubert, Proust e Beckett (per il quale ho un vero e proprio culto), Kierkegaard, Wallace Stevens e Marianne Moore, Paul Klee, gli scritti di Mondrian. Fra i poeti contemporanei è stato importantissimo Philippe Jaccottet.

Oggi sento il bisogno di contenere l'attrazione per i russi, ma anche per i mistici: da Giovanni della Croce a Maria Zambrano. Mi sono riavvicinata alla poesia in lingua inglese, ma non amo la poesia confessionale americana. Da qualche tempo ho riletto con attenzione Elizabeth Bishop (che, infatti, prende le distanze dalla poesia confessionale). Capita di leggere un poeta, di ammirarlo magari, ma non di "vederlo" davvero. Mi è capitata la stessa cosa con Pascoli.

Hai affermato che la poesia sostituisce talvolta una stretta di mano, che è atto di comunicazione, un ritrovarsi con l'altro. Ma il poeta è solo quando scrive e spesso è solo perché non può stare dove si compiono i riti della volgarizzazione della vita e anche della 
morte. La solitudine è una condizione e la poesia è il ponte per uscirne?

"La poesia come una stretta di mano" è un'affermazione di Paul Celan, altro poeta per me importante che mancava all'elenco. Questo però non esclude la solitudine che è una condizione non necessariamente negativa, almeno per me. Anzi.

Il "nome è anche raggiungere se stessi", hai scritto in una poesia. Ma allo stesso tempo conviviamo con l'appiattimento della lingua, con nomi che non significano, che non riportano più né alle cose né agli esseri, talmente sono logori e generici. Può il poeta, da solo, reinaugurare la lingua, rifondare un nuovo rapporto fra il nome e le cose?

Il verso "se nome è anche raggiungere se stessi" mi è stato suggerito da una riflessione di Giacoma Limentani nel suo libro Il Midrash, quando nota che in ebraico Shem (nome) e Sham (luogo, ma nel senso di andare verso un luogo, moto a luogo) hanno la stessa radice. Raggiungere il proprio nome come se fosse un luogo è mettersi in cammino verso se stessi, non solo diventare se stessi, ma smettere di essere ciò che si era accettando di attraversare la propria aridità, il proprio deserto. Non penso però che il poeta debba o possa "rifondare un nuovo rapporto tra il nome e le cose". Le cose sono qui e le parole possono essere logore, anzi "logoro" è una parola molto bella. L'importante è provare a non essere generici, ma questo è un problema di attenzione, di sforzo. C'è la tentazione a volte di lasciar perdere, di abbandonarsi al suono della propria poesia, alla genericità (che è anche abilità).

In un verso hai scritto: "dai forma al buio". è quello che resta al poeta di oggi? Perscrutare, modellare, ordinare il buio?

Intendevo proprio un gesto concreto. Plasmare il buio come una materia, come si fa nella scultura. Alla poesia "restano" in realtà molte cose, ma le fa guardando, pensando, ascoltando e scrivendo e riscrivendo.

Ogni poeta ha un concetto di poesia, ne dà una diversa definizione. Per te, che cos'è la poesia?

Una cosa terrena, un dono e un lavoro, provando ad andare avanti e invece magari tornando indietro. Insomma un fare molto precario, come la vita.

Scrivi spesso? Lo fai metodicamente, come tanti poeti? $O$ per te la poesia è quel lampo, quell'illuminazio però che sia fra i coetanei che fra i più giovani ci siano molte brave poetesse 
e molti bravi poeti. L'elenco sarebbe lungo, ma comprenderebbe orientamenti diversi senza recinti di scuole. M'interessa moltissimo la poesia dialettale, la leggo come leggerei una poesia straniera, ma forse essendo di origini sardo-corse leggo anche gli italiani così. 